\title{
La répartition des affaires entre les juges
}

Cette contribution étudie la pratique suisse de la répartition des affaires entre les juges au niveau fédéral et cantonal à l'exemple des cantons romands et du Tessin. En laissant un large pouvoir de discrétion à chaque tribunal, la pratique suisse s'oppose à la jurisprudence de la CourEDH qui requiert un encadrement de ce pouvoir de répartition des affaires. Le TF, ayant nouvellement qualifié cette pratique de "solution transitoire ", ainsi que les autres instances fédérales font figures de bons élèves en se dotant d'un règlement encadrant mieux la répartition des affaires entre les juges, encourageant les tribunaux suisses à adapter leur règlement interne.

Catégories d'articles : Forum

Proposition de citation : Fabian Teichmann / Madeleine Camprubi / Léonard Gerber, La répartition des affaires entre les juges, in : «Justice - Justiz - Giustizia» 2020/4 


\section{Table des matières}

Introduction

I. Le droit à une composition régulière du tribunal appelé à statuer

II. La position du Tribunal fédéral

1. Général

2. Pouvoirs de discrétion des juges pour l'attribution des procédures

III. Règlements des instances fédérales

IV. Règlements des tribunaux cantonaux en Romandie et au Tessin

V. Pratique de la CourEDH

Conclusion

\section{Introduction}

[1] Dans l'accomplissement de ses tâches juridictionnelles, la justice doit être indépendante nonseulement à l'égard des autres pouvoirs mais également, entre autres, à l'abri de l'influence des partis politiques. ${ }^{1} \mathrm{Il}$ en va de l'indépendance institutionnelle de la justice et personnelle du juge selon l'art. 30 I Cst.féd. ${ }^{2}$ et l'art. $6 \S 1$ CEDH. Or, l'on ne peut exclure d'avance une certaine influence des juges de la part de leur orientation politique, de leur ressenti personnel par rapport à une affaire précise ou même de conflits d'intérêts avec ceux des parties à la procédure. Le droit constitutionnel à une composition régulière du tribunal et les règles de récusation ont pour but de corriger ce hasard moral.

[2] Cette contribution comprend un bref rappel du droit à la composition régulière du tribunal et décrit l'état de la jurisprudence ainsi que les exigences en cette matière au niveau du TF et de la CourEDH, plus particulièrement quant à la réparation des affaires entre les juges. Il examine dans un deuxième temps les règlements des tribunaux cantonaux romands et des implications pratiques de la jurisprudence du TF et de la CourEDH ainsi que de l'opportunité d'un recours auprès de la CourEDH.

\section{Le droit à une composition régulière du tribunal appelé à statuer}

[3] Conformément à l'art. 30 I Cst.féd. et $6 \S 1 \mathrm{CEDH}$, toute personne a droit à ce que « sa cause soit jugée par un tribunal indépendant, impartial et établi par la loi ». ${ }^{3}$ Cette garantie a pour but d'empêcher que la jurisprudence soit influencée par une sélection insidieuse des juges formant l'autorité décisionnelle. ${ }^{4}$ La garantie de la composition régulière de l'autorité judiciaire comporte alors deux aspects. ${ }^{5}$ D'une part, l'autorité collégiale doit être formée " comme la loi le prévoit ", notamment s'agissant des qualités des membres ou du quorum, et, d'autre part, en respect de l'impartialité de l'autorité appelée à statuer pouvant justifier une récusation. ${ }^{6}$

\footnotetext{
Voir par exemple Etienne Poltier, L'organisation et le fonctionnement interne de l'ordre judiciaire et des tribunaux, PJA 2011, p. 1018-1036, p. 1018.

2 Voir Poltier (n. 1), p. 1018.

3 Voir également l'art. $14 \S 1$ du Pacte ONU II encadrant « l'accusation pénale » et « les contestations sur des droits et obligations de caractère civil ».

4 Voir notamment l'ATF 137 I 340, c. 2.2.1. ; Voir également Poltier (n. 1), p. 1019.

5 Thierry Tanquerel, Manuel de droit administratif, 2ème éd., Genève 2018, p. 510-539, p. 515.

6 TANQuerel (n. 5), p. 515.
} 
[4] Les règles de récusation visent à exclure tout conflit d'intérêts entre les parties et l'autorité appelée à statuer et concrétisent ainsi la garantie constitutionnelle d'impartialité du tribunal. ${ }^{7}$ Des doutes sur l'impartialité d'un juge fondés sur des critères objectifs notamment liés à son comportement ou à sa situation suffisent à transgresser cette garantie d'impartialité, un abus concret n'est pas requis. ${ }^{8}$

[5] En revanche, les parties peuvent se retrouver incapables de lutter contre la situation où le collège de juges appelé à statuer n'est formé que de "hardliners", démontre une plus grande " sévérité » que d'autres, lorsque les membres d'un parti politique sont surreprésentés ou lorsque la situation exigerait par exemple une représentation paritaire des deux genres. Dans ces situations, la garantie de la composition régulière du tribunal tire particulièrement son sens lorsqu'il n'existe aucun motif de récusation.

\section{La position du Tribunal fédéral}

\section{Général}

[6] Les lignes directrices auxquelles se tient actuellement le $\mathrm{TF}^{9}$ disposent ce qui suit : « La composition du collège appelé à statuer devrait être réglée en principe de façon abstraite et générale. Il est indispensable qu'elle apparaisse comme un acte de l'autonomie administrative de la justice et ne soit pas soumise à l'influence de l'exécutif ». Le collège réunit les juges chargés de statuer sur une cause, ainsi un président et des juges ordinaires. Ce collège doit statuer in corpore sur l'ensemble des causes dont il est saisi. ${ }^{10}$ Deux solutions principales se présentent. D'une part, celle d'une désignation automatisée suivant le tour de rôle, et celle d'une attribution par le président du tribunal. ${ }^{11}$ Dans tous les cas, le président du tribunal n'a pas de pouvoir d'instruction dans le traitement de l'affaire. ${ }^{12}$ L'art. 30 I Cst.féd. n'exclut toutefois pas un certain pouvoir d'appréciation dans la composition du collège appelé à statuer, "aussi longtemps que celle-ci est prévue par la loi et repose sur des critères déterminés d'avance pour chaque cas d'espèce à prendre en considération, et objectifs qui permettent un traitement approprié $d u$ cas dans un délai convenable $" .{ }^{13}$ Cette dernière formule est centrale. Elle octroie au représentant de la justice la compétence d'organiser la composition sur la base de critères variés (comme la charge de travail, la langue, le taux d'occupation ou des connaissances spécifiques $)^{14} \cdot{ }^{15}$ Le TF s'est quant à lui doté d'un tel règlement ${ }^{16}$. Il en va

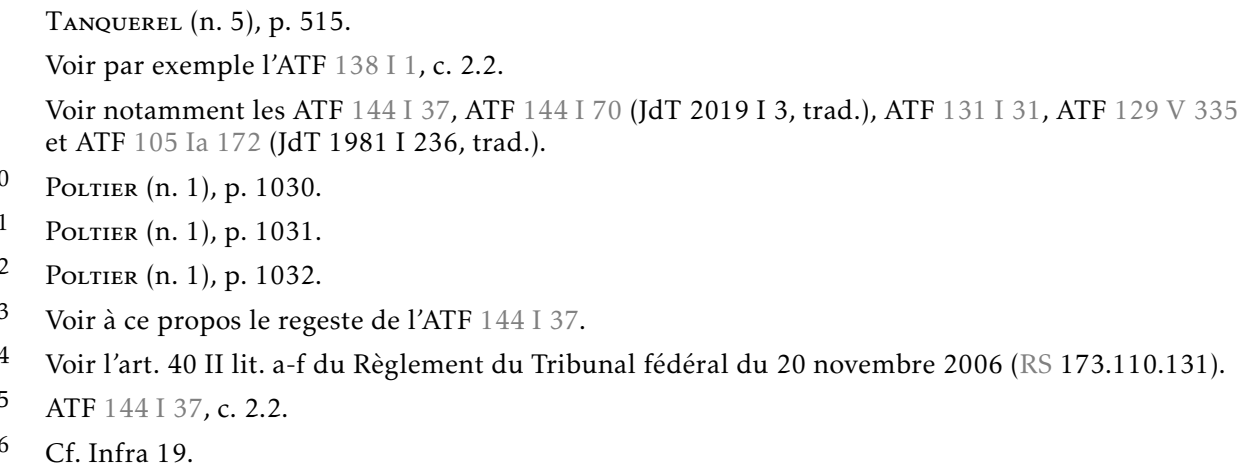


de même du Tribunal pénal fédéral ${ }^{17}$ ainsi que du Tribunal administratif fédéral ${ }^{18}$. La doctrine est toutefois partagée s'agissant de l'étendue du pouvoir de discrétion des tribunaux quant à la répartition des affaires entre les juges. ${ }^{19}$ Une partie de la doctrine plaide pour une restriction forte ${ }^{20}$ du pouvoir de discrétion tandis que d'autres auteurs encouragent la flexibilité. ${ }^{21}$

[7] Cette pratique se justifie sur la base d'une théorie présentée par le TF en 1979 selon laquelle l'attribution aveugle des procédures individuelles entre les juges représenterait une réflexion théorique de la justice idéale. ${ }^{22}$ Toutefois, une application concrète d'une telle théorie dans la pratique ne serait pas possible. ${ }^{23}$ On se demandera cependant si un pouvoir décisionnel discrétionnaire quant à la composition du collège chargé d'une affaire se justifie encore à la vue des développements technologiques actuels. Le TF relève dans l'ATF 144 I 70 la critique correspondante de la doctrine ${ }^{24}$, sans toutefois modifier sa pratique. Il argumente que sa pratique constitue un compromis entre les avantages et désavantages d'une composition schématique du collège appelé à statuer. ${ }^{25} \mathrm{Il}$ souligne également que le pouvoir de discrétion dans la composition du collège doit être encadré par des critères matériels et transparents. ${ }^{26}$ Les instances judiciaires cantonales dont la composition des collèges appelés à statuer présentant les mêmes standards que les organes de la justice au niveau fédéral devraient en principe remplir les exigences de l'art. 30 I Cst.féd. Aussi longtemps qu'aucune circonstance ne sorte de l'ordinaire ${ }^{27}$, des critères objectifs déterminés d'avance et basés sur un règlement ou une pratique transparente suffiront.

[8] Il est à noter que le pouvoir de cognition du TF lorsqu'il interprète le droit cantonal est limité à l'arbitraire. ${ }^{28}$ Cependant, le TF a légèrement modifié sa définition de l'arbitraire, à notre sens plus restrictive. Il ressort que malgré le pouvoir de cognition du TF limité à l'arbitraire lorsqu'il traite de l'application du droit cantonal, le TF dispose d'un pouvoir d'examen libre lorsqu'il examine si l'interprétation et l'application du droit cantonal sont compatibles avec la garantie constitution-

17 Règlement sur l'organisation du Tribunal pénal fédéral du 31 août 2010 (RS 171.713.161).

18 Règlement du Tribunal administratif fédéral du 17 avril 2008 (RS 173.320.1). Voir notamment l'art. 15 ROTPF respectivement les art. 31 et 32 RTAF.

19 Regina Kiener, Anforderungen des Europarats und der OSZE an die Spruchkörperbildung in Gerichten, in : Michel Hottelier/Maya Herrtig Randall/Alexandre Flückiger (éds.), Études en l’honneur du Professeur Thierry Tanquerel, CG, Genève 2019, p. 193-199, p. 193 ss.

20 Voir par exemple, Jörg Paul Müller/Markus Schefer, Grundrechte in der Schweiz, 4ème éd., Berne 2008, p. 934 s., Arnold Marti, Kommentar zu den beiden Bundesgerichtsurteilen zur Gerichtsbesetzung in den Kantonen Bern und Basel-Stadt, ZBl 119/2018, p. 357 ss., p. 362, UlRICH Cavelti, Der Gerichtspräsident - primus inter pares oder Justizmanager?, in : Peter Mäusli-Allenspach/Michael Beusch (éds.), Steuern und Recht - Steuerrecht!, Liber amicorum für Martin Zweifel, Bâle 2013, p. 325 ss., p. 331

21 Voir par exemple Hansjörg SeILER, Bundesgerichtsgesetz (BGG) - Handkommentar, 2ème éd., Berne 2015, art. 22 BGG N 7 f., Thomas Müller-Graf, «Sehende» Richter braucht das Land, in : Justice - Justiz - Giustizia 2009/4, Rz. 1, ainsi que Hans Peter Walter, Interne richterliche Unabhängigkeit, in : Justice - Justiz - Giustizia 2005/1, Rz. 17. ATF 105 Ia 172, c. 2 (JdT 1981 I 236, c. 2, trad.).

27 Voir notamment l'ATF 105 Ia 172 (JdT 1981 I 236, trad.) ainsi que l'arrêt du Tribunal fédéral 6P.102/2005 du 26 juin 2006.

28 Voir notamment l'ATF 129 V 335, c. 1.3. ; 126 V 303, c. 1a ; 137 I 340, c. 2.1 et 2.2. 
nelle d'un tribunal établi par la loi compétent indépendant et impartial sur la base de l'art. 30 I Cst.féd. ${ }^{29}$

\section{Pouvoirs de discrétion des juges pour l'attribution des procédures}

[9] Lorsque le pouvoir de discrétion du président de l'instance judiciaire quant à la composition du collège appelé à statuer n'est limité par aucun critère objectif, ceci n'implique pas nécessairement une violation de l'art. 30 I Cst.féd., selon la pratique du TF. ${ }^{30}$ Le TF a en effet sauvé cette possibilité en considérant qu'il n'y avait pas de violation de l'art. 30 I Cst.féd. dans cette situation. De plus, il relève dans l'ATF 144 I 70 que la jurisprudence européenne soutient également "l'importance d'une désignation des juges fondés sur des règles, sans toutefois qu'une base légale » soit nécessaire du moment que « les critères abstraits soient définis à l'avance et de manière transparente " sur la base d'une pratique du tribunal. ${ }^{31}$ Il n'est pas non-plus exigé que "tout pouvoir d'appréciation soit exclu mais que la désignation des juges soit encadrée par des critères matériels $» .^{32}$

[10] Sur la base de ces développements devrait-on penser que la désignation des juges par le président se basant exclusivement sur des critères de désignation établis par la pratique viole l'art. 30 I Cst.féd. respectivement l'art. $6 \mathrm{CEDH}$ ? Il est difficilement concevable comment un sujet de droit peut prendre connaissance de ces critères lorsqu'ils ne font pas l'objet d'une norme générale et abstraite, d'autant plus que la méthode de désignation des juges ne sera ni comprise dans le jugement, ni communiquée préalablement aux parties. ${ }^{33}$ Ce problème est toutefois adressé par le TF qui statue que la définition de ces critères peut également être le résultat d'une " pratique bien établie " comme règle générale et abstraite. ${ }^{34}$ Dans l'affaire concrète toutefois, le TF ne retient aucune pratique bien établie de la part du Tribunal cantonal bernois, mais un règlement cantonal selon lequel la répartition des affaires entre les juges est fixée selon la charge de travail. Le TF considère qu'il s'agit d'un critère objectif et a donc rejeté une violation de l'art. 30 I Cst.féd. ${ }^{35}$

[11] Reste non-répondue la question dans quelle mesure cette méthode de désignation des juges permet d'éviter une apparence de manipulation de la part de l'instance judiciaire. En effet, le critère avancé de la charge de travail laisse place à une quantité de compositions différentes, n'excluant donc aucunement des compositions propres à favoriser des décisions dans une direction voulue par le président de la cour en question. On relèvera cependant que ce n'est que par obiter dictum que le TF soutient ces pratiques bien établies de désignation des juges comme base pour la composition de la cour. Le TF est vraisemblablement animé par un souci de sécurité juridique et par la protection de la confiance des justiciables au système judiciaire, si bien qu'il se réserve de remettre en cause les pratiques des tribunaux encore courantes des cantons. Il est impossible de

29 Cf. ATF 131 I 31, c. 2.1.1. ; ATF 127 I 128, c. 3c. Voir aussi Fabian Teichmann/Madeleine Camprubi, Der Anspruch auf rechtmässige Spruchkörperbesetzung nach Art. 30 BV, forumpoenale 1/2020, p. 42-47, p. 43.

30 Une telle situation est répandue parmi les cantons.

31 ATF 144 I 70, c. 5.6, (JdT 2019 I 3, c. 5.6., trad.).

32 Arrêt du Tribunal fédéral 1C_187/2017 du 20 mars 2018, c. 6.6., voir également JdT 2019 I 3 (trad.)

33 Cf. ATF 144 I 70, c. 4.4. (JdT 2019 I 3, c. 4.4., trad., dans lequel la présidente de la Chambre de recours pénale a déjà offert des explications à l'avocat de la recourante quant à la composition de la cour appelée à statuer.

34 ATF 144 I 70, c. 5.6. (JdT 2019 I 3, c. 5.4. (trad.) « leur définition pouvant aussi résulter d'une pratique bien établie ». Voir également l'arrêt du Tribunal fédéral 6B_63/2018 du 21 juin 2018, c. 3.2.1.

35 ATF 144 I 70, c. 6. (JdT 2019 I 3, c. 6. (trad.) confirmé par l'arrêt du Tribunal fédéral 1B_546/2017 du 11 mai 2018. 
déterminer jusqu'à quand le TF continuera de protéger la répartition discrétionnaire des affaires entre les juges lorsqu'elle repose uniquement sur des pratiques bien établies et non sur une règle générale et abstraite. Les cantons et les autorités judiciaires sont ainsi avisées de modifier leur pratique, au minimum de fixer par voie réglementaire des critères objectifs permettant d'exercer leur pouvoir de discrétion dans la répartition des affaires entre les juges. À cet égard, plus le pouvoir discrétionnaire sera limité, le mieux répondront-ils à l'obiter dictum du TF.

[12] Suite à l'ATF 144 I 70, une autre cour du Tribunal fédéral a relevé dans une autre affaire que la réglementation de la répartition des affaires entre les juges, concernant également le Tribunal cantonal bernois, s'avère problématique. ${ }^{36}$ Dans cet arrêt, le TF soulève que mis à part le critère de la répartition de la charge de travail, il n'existe aucun critère abstrait, transparent et défini à l'avance limitant matériellement la discrétion du président de section. Conformément à la position adoptée dans l'ATF 144 I 70, le TF accepta cependant de justesse la composition du Tribunal cantonal bernois, faisant valoir que son règlement d'organisation suffisait en tant que "solution transitoire $»{ }^{37}$ Par ailleurs, il renonça aux frais de justice dans cette même affaire, justifiant ainsi de fait le fonds du recours. ${ }^{38}$ Un autre indice indiquant qu'il ne s'agit que d'une question de temps avant que le TF ne rejette les pratiques des tribunaux en matière de répartition des affaires entre les juges non basées sur une norme générale et abstraite, est la référence subséquente aux recommandations de la commission de Venise dans l'ATF 144 I 70. Ces dernières enjoignent vivement que les critères réglant l'attribution des affaires entre les juges soient transparents, objectifs et établis à l'avance par la loi ou par un règlement spécial fondé sur la loi, comme le règlement du tribunal. ${ }^{39}$

[13] Cet arrêt contraste avec la jurisprudence plus ancienne du TF remontant à un arrêt de 1979, dans lequel il relevait que la pratique suisse impliquerait une répartition des affaires entre les juges laissée à la discrétion du président du tribunal. ${ }^{40} \mathrm{Il}$ remarquait également qu'aucune tendance politique à vouloir modifier cette pratique ne se ferait ressentir dans les cantons. ${ }^{41} \mathrm{Il} \mathrm{sou-}$ lignait la doctrine allemande dissidente qui implique que le pouvoir de discrétion du président devait être restreint autant que possible avec des tours fixés à l'avance. ${ }^{42}$ Une telle répartition à l'aveugle relèverait cependant d'une "représentation théorique d'une justice idéale ${ }^{43}$ Elle conduirait à des problèmes pratiques, raison pour laquelle le TF s'était distancé d'une telle position. Ainsi, elle empêcherait le tribunal de faire appel à l'expertise particulière de certains juges suppléants dans des domaines spéciaux, par exemple en droit fiscal, en droit de la construction ou

36 Cf. Arrêt du Tribunal fédéral 1B_63/2018 du 21 juin 2018, ainsi que l’arrêt du Tribunal fédéral 6B_1458/2017 du 21 juin 2018, c. 3.2.3.

37 Arrêt du Tribunal fédéral 6B_1458/2017 du 21 juin 2018, c. 3.2.3.

38 Arrêt du Tribunal fédéral 1B_63/2018, du 21 juin 2018, c. 4. ainsi que le dispositif aux points 1 et 2; Voir également l'art. 66 LTF.

39 ATF 144 I 70, c. 5.4. (JdT 2019 I 3, c. 5.4. (trad.) avec références au rapport de la commission de Venise du 16 mars 2010, Etude ${ }^{\circ} 494 / 2008$.

$40 \quad$ ATF 105 Ia 172, c. 5b (JdT 1981 I 236, trad.).

41 ATF 105 Ia 172, c. 5b (JdT 1981 I 236, trad.)

42 ATF 105 Ia 172, c. 5b (JdT 1981 I 236, trad.), ainsi que les références citées.

43 ATF 105 Ia 172, c. 5b (JdT 1981 I 236, trad.). 
en droit commercial. ${ }^{44}$ De plus, elle surchargerait le travail des juges ordinaires et augmenterait la durée moyenne des procès, préjudiciable pour la justice. ${ }^{45}$

\section{Règlements des instances fédérales}

[14] Les instances fédérales font figure de bonnes élèves en prévoyant des critères objectifs nécessaires à la répartition de chaque affaire entre les juges directement au niveau règlementaire. Les règlements respectifs du TF et du TPF prévoient que le président de chaque cour compétente est responsable de constituer le collège appelé à statuer. ${ }^{46}$ Quant au TAF, l'attribution des affaires s'effectue selon une clé de répartition par programme informatique (Bandlimat), fixée à l'avance par la cour selon l'ordre d'entrée des affaires. ${ }^{47}$ Que la réparation se fasse par clé ou selon la discrétion du président de la cour, chaque règlement prévoit qu'il est tenu compte de "l'équilibre de la charge de travail des juges, de la langue officielle, de la participation des membres des deux sexes, des connaissances spécifiques des juges dans un domaine déterminée ainsi que des absences dues aux vacances ou d'une malade dans la répartition des affaires ».

\section{Règlements des tribunaux cantonaux en Romandie et au Tessin}

[15] La grande majorité des règlements des tribunaux cantonaux romands prévoit que la répartition des affaires entre les juges demeure à la discrétion du président du Tribunal cantonal ou de la cour concernée. Il en va ainsi des membres présidents de chaque section du Tribunal cantonal du Jura (art. 26 I LOJ-JU et art. 12 du Règlement du Tribunal cantonal jurassien), du membre président de chaque cour du Tribunal cantonal de Neuchâtel (art. 18 let. a du Règlement du Tribunal cantonal neuchâtelois), de la présidence du Tribunal cantonal du Valais (art. 26 I ROT-VS), de la présidence du Tribunal cantonal fribourgeois (art. 35 I RTC-FR) et de la Cour de justice du canton de Genève à défaut de décharge de la présidence (art. 29 IV let. a LOJ-GE et art. 9 I RCJ-GE). Il en va de même pour le canton du Tessin où le membre président de la section du Tribunal d'appel attribue les affaires (art. 22 du règlement du Tribunal d'appel). Le canton de Vaud quant à lui attribue au greffe du Tribunal cantonal la faculté d'attribuer les procédures entre les juges, sous réserve de dérogation du président de la cour concernée (art. 42 II et III ROTC-VD). Les cantons romands optent donc majoritairement pour une répartition des affaires laissées à la discrétion des tribunaux concernés.

[16] Ce pouvoir de discrétion dans la répartition des affaires est rarement encadré par des critères objectifs au niveau légal ou réglementaire. Les règlements des tribunaux cantonaux doivent donc être précisés par le biais de décisions internes soit d'une pratique interne propre à chaque tribunal ou cour à laquelle souvent le justiciable n'a pas accès. Le Tribunal d'appel tessinois prévoit que la répartition a lieu selon l'ordre chronologique d'arrivée des affaires, à défaut de cause urgente (art. $14 \mathrm{I}$ du règlement du Tribunal d'appel). Dans le canton du Jura, la répartition des

44 ATF 105 Ia 172, c. 5b (JdT 1981 I 236, trad.).

45 ATF 105 Ia 172, c. 5b (JdT 1981 I 236, trad.).

46 Voir l'art. 40 RTF et l'art. 15 ROTPF.

47 Art. 31 III RTAF. 
affaires a lieu selon un consensus à la discrétion totale du ou des membres présidents de chaque section, voire du plénum du Tribunal cantonal (art. 12 III et 13 II du Règlement du Tribunal cantonal jurassien). À Neuchâtel ainsi qu'au Valais, le président répartissant les affaires veille à une répartition équilibrée des charges (art. 18 let. a Règlement du Tribunal cantonal Neuchâtelois et art. 26 I let. d ROT-VS). Cette dernière situation semble toutefois similaire au cas du Tribunal cantonal bernois que le TF qualifia de "solution transitoire ". ${ }^{48}$ À Fribourg, la répartition des affaires s'effectue selon le principe aléatoire à moins que le président décide d'une modification si nécessaire, sans donner plus de précision au niveau règlementaire (art. 35 I RTC-FR). Il est toutefois tenu compte de la représentation des deux langues officielles au sein des autorités judiciaires (art. 20 I et 115 III LJ-FR), si bien qu'il est vraisemblablement aussi tenu compte de la langue de la procédure lors de l'attribution. Dans le canton de Genève, les cours composant la Cour de justice du canton de Genève "s'organisent elles-mêmes » (art. 25 I LOJ-GE et art. 18 I RCJ), il est tenu compte de l'expérience acquise, des compétences particulières dans les branches du droit, voire de l'équilibre des sensibilités politiques dans la répartition des juges dans la composition des chambres (art. 118 II LOJ-GE et art. 22 RCJ). Toutefois, ni la RCJ ni la LOJ-GE ne semblent encadrer le pouvoir de discrétion de la présidence. En revanche, seul le canton de Vaud encadre la faculté du greffe de son Tribunal cantonal avec des critères objectifs directement au niveau règlementaire (art. 42 II ROTC-VD). Ainsi, le greffe doit en premier lieu appliquer un tournus déterminé par l'ordre d'élection de chaque juge (art. 60 ROTC-VD) tout en tenant compte d'autre part du taux d'activité des juges. Le membre président de la cour peut déroger à la répartition du greffe lorsque de justes motifs liés notamment à l'équilibre de la charge de travail des juges, des absences et de la distribution selon le genre en fonction de la nature du litige (art. 42 III ROTC). La répartition des procédures a donc lieu selon le critère de l'ordre d'élection des juges $\mathrm{du}$ Tribunal cantonal vaudois.

[17] Par conséquent, les tribunaux cantonaux sont enjoints de fixer au niveau réglementaire des critères objectifs et transparents encadrant la répartition des affaires entre les juges. La majorité des règlements des tribunaux cantonaux romands et tessinois, à l'exception de celui du Tribunal cantonal vaudois renvoie au pouvoir de discrétion des membres de leurs cours à l'instar du Tribunal cantonal bernois en 2018.

[18] Il serait donc opportun que les tribunaux mentionnés adaptent leurs règlements de manière à se conformer à la jurisprudence pour éviter le risque d'être qualifié de "solution transitoire ». Ceci est d'autant plus indiqué à la vue de la jurisprudence de la CourEDH dont il est question au chapitre suivant et des règlements adoptés par les instances judiciaires fédérales. Invoquer une violation du droit à une composition régulière du tribunal en raison de la composition viciée de la cour appelée à statuer présente des chances de succès, surtout si la désignation du collège statué à juger repose uniquement sur le pouvoir discrétionnaire non-limité ou quasi non-limité du tribunal. En effet, le TF n'a pas de raison de s'écarter prochainement de ses standards jurisprudentiaux, et il se peut bien qu'il se montre plus strict à l'avenir quant à la constitution du collège de juges basée certes sur une pratique constante, mais sans ancrage dans la loi ou tout du moins, le règlement du tribunal. Enfin, il est à rappeler qu'une violation de l'art. 30 I Cst.féd. conduit à la nullité totale de la décision, indépendamment d'un effet de ce vice sur le résultat du jugement. ${ }^{49}$

48 Voir l'arrêt du Tribunal fédéral 6B_1458/2017 du 21 juin 2018, c. 3.2.2s.

49 ATF 137 I 340, 342 ; arrêt du Tribunal fédéral 1C_85/2014 du 9 avril 2015, c. 2.7. Cette garantie s'applique plus spécialement par le biais de l'art. 4 CPP, voir à cet égard Alain Macaluso/Gérard Piquerez, Procédure pénale, 
[19] Les standards du TF n'enterrent pas complètement le problème de la sélection ciblée des juges pour une affaire. Une limitation plus forte du pouvoir discrétionnaire des tribunaux notamment en le combinant avec un algorithme aléatoire, comme c'est déjà le cas au Tribunal administratif fédéral ${ }^{50}$, ou en le remplaçant entièrement par un tel procédé, par exemple en répartissant chaque affaire par date de réception ou par ordre alphabétique permettront certainement de limiter ce risque. ${ }^{51}$

\section{Pratique de la CourEDH}

[20] Les garanties de l'art. 6 CEDH sont applicables non seulement à la composition, l'organisation et la compétence des instances judiciaires, mais également à l'attribution des affaires aux juges devant statuer sur une affaire. ${ }^{52} \mathrm{La}$ CourEDH démontre une certaine réticence quand elle examine l'application du droit national. ${ }^{53}$ La CourEDH ne s'est jamais explicitement prononcée quant à l'exactitude des normes générales et abstraites devant régir la répartition des affaires entre les juges.

[21] Sur la base de l'art. $6 \mathrm{CEDH}$, une composition de la cour se basant uniquement sur le pouvoir d'appréciation d'un membre du tribunal demeure en tout cas problématique. Il en va de même lorsque le pouvoir d'appréciation n'est que rudimentairement encadré par une norme générale et abstraite. La CourEDH en vient à cette même conclusion dans l'affaire Miracle Europe Kft v. Hongrie le 12 janvier 2016. ${ }^{54}$ Dans cette procédure, la CourEDH statua que la réattribution d'une affaire par une autorité exécutive à une instance judiciaire autre que le tribunal de première instance territorialement compétente ne reposait sur aucune base légale ou matérielle, et violait ainsi l'art. $6 \mathrm{CEDH} .{ }^{55}$ Elle relève l'importance de considérer l'ensemble des circonstances du cas concret, ${ }^{56}$ et rappelle obiter dictum les risques d'abus liés à une répartition des affaires sur la base de la discrétion des tribunaux. ${ }^{57}$ Elle indique cependant aussi qu'une interprétation restrictive du droit à un tribunal établi par la loi ne se justifie pas. ${ }^{58}$ Ainsi, la CourEDH n'exclut pas

3ème éd., Genève 2011, p. 140-169, p. 152. Il en va de même pour l'art. 6 § 1 CEDH (voir à ce propos CourEDH, 12 avril 2018, Chim and Przywicreski c. Pologne, n³6661/07 et 38433/07 § 217).

50 Voir l'art. 31 III RTAF selon lequel « l'attribution des affaires au sens de l'al. 2 s'effectue selon une clé fixée à l'avance par la cour. Cette clé est soumise à l'approbation de la Commission administrative. L'ordre d'entrée des affaires est déterminant pour la fixation de la clé. Celle-ci doit également tenir compte de manière adéquate des langues officielles, du taux d'occupation des juges et de la charge de travail occasionnée par leur participation à des organes du tribunal, ainsi que d'autres critères, comme par exemple les compétences spécifiques d'une chambre ou du fait qu'un juge s'est déjà occupé d'une affaire. ».

51 Voir notamment Regina KIENER, Richterliche Unabhängigkeit, Bern 2001, p. 376 ss.; Voir également les références dans l'ATF 144 I 70, c. 5.2.

52 Jens Meyer-Ladewig/Stefan Harrendorf/Stefan König, in : Jens Meyer-Ladewig/Martin Nettesheim/Stefan von Raumer (éds.), Handkommentar Europäische Menschenrechtskonvention, 4ème éd., Bâle 2017, art. 6 N 71. Voir également les références de l’ATF 144 I 70, c. 5.2 s. Voir également CourEDH, 12 janvier 2016, Miracle Kft c. Hongrie, $n^{\circ} 57774 / 13 \S 50$. CourEDH, 12 janvier 2016, Miracle Kft c. Hongrie, n57774/13. CourEDH, 12 janvier 2016, Miracle Kft c. Hongrie, n57774/13 §§ 61 ss., voir plus particulièrement $\S 63$, dans lequel «neither ascertainable reasons nor criteria as to which cases were to be transferred ».

56 CourEDH, 12 janvier 2016, Miracle Kft c. Hongrie, ${ }^{\circ} 57774 / 13 \S 46$ postulant que « it takes into account the proceedings as a whole ».

57 CourEDH, 12 janvier 2016, Miracle Kft c. Hongrie, n57774/13 58.

58 CourEDH, 12 janvier 2016, Miracle Kft c. Hongrie, nº57774/13 45 avec les références citées. 
de manière absolue tout pouvoir de discrétion dans la répartition des affaires entre les juges ${ }^{59} \mathrm{si}$ bien que les règlements comme celui du Tribunal fédéral ne devraient pas causer de problème face à la jurisprudence de la CourEDH. Par contre, au vu de la jurisprudence de la CourEDH et de l'obiter dictum du TF, la répartition des affaires entre les juges d'un tribunal doit être encadrée de façon transparente par des critères objectifs.

[22] Un recours individuel selon les art. 34 ss. CEDH auprès de la CourEDH reste ouvert pour violation de l'art. $6 \S 1 \mathrm{CEDH},{ }^{60}$ après épuisement des voies de recours nationaux (art. $35 \S 1$ $\mathrm{CEDH})$. Il suffira que le recourant ait invoqué par exemple l'art. 30 I Cst.féd. puisqu'il correspond dans son essence à l'art. $6 \mathrm{CEDH} .{ }^{61}$ Comme le vice est formel, il engendre la nullité de la décision en question, qu'il ait ou non un impact sur le résultat final, ${ }^{62}$ constituant ainsi un préjudice important au sens de l'art. $35 \S 3$ lit. b CEDH. ${ }^{63}$

\section{Conclusion}

[23] La pratique dans les tribunaux cantonaux romands est à la délégation de la faculté d'attribuer les affaires entre les juges à la discrétion de la présidence du tribunal, de la cour voire du greffe en question. Cette discrétion n'est que rarement encadrée par des critères objectifs transparents au niveau réglementaire et fait toujours l'objet de pratiques internes aux tribunaux opaques pour le justiciable. Seul le Tribunal cantonal vaudois s'est doté d'un règlement comprenant de pareils critères objectifs. Il est difficile de dire jusqu'à quand le TF protègera ces pratiques cantonales avant qu'il ne décide de renverser ce qu'il qualifie de « solution transitoire » à l'exemple du règlement du Tribunal cantonal bernois. Cependant, au vu de la jurisprudence de la CourEDH, du TF et des règlements adoptés par les instances judiciaires fédérales, il y a de fortes probabilités que le TF se montre plus strict, ce qui augmenterait les perspectives de succès de voies de recours contre les décisions prises par les collèges de juges en question, à moins que les tribunaux concernés n’y remédient à temps.

Fabian Teichmann, Dr. iur. Dr. rer. pol., LL.M., Rechtsanwalt, Teichmann International (Schweiz) AG.

Madeleine CAmprubi, PD Dr. iur, Rechtsanwältin, Teichmann International (Schweiz) AG.

LÉONARD Gerber, BLaw, Teichmann International (Schweiz) AG.

59 CourEDH, 12 janvier 2016, Miracle Kft c. Hongrie, n57774/13§51 avec les références citées.

60 Cf. art. 34 ss. CEDH.

61 Jens Meyer-Ladewig/Peter Szczekalla, in : Jens Meyer-Ladewig/Martin Nettesheim/Stefan von Raumer (éds.), Handkommentar Europäische Menschenrechtskonvention, 4ème éd., Bâle 2017, art. 35 N 9 ; PAtrick Schäfer, in : Ulrich Karpenstein/Franz Mayer (éds.), Konvention zum Schutz der Menschenrechte und Grundfreiheiten, Kommentar, 2ème éd., Munich 2015, art. 35 N 11.

62 Voir à ce propos CourEDH, 12 avril 2018, Chim and Przywicreski c. Pologne, nº36661/07 et 38433/07§ 217.

63 Cf. art. $35 \S 1 \mathrm{CEDH}$. 